\title{
A Paper-Digital Interface for Information Capture and Display in Time-Critical Medical Work
}

\author{
Aleksandra Sarcevic ${ }^{1}$, Nadir Weibel ${ }^{2}$, James D. Hollan ${ }^{2}$, Randall S. Burd ${ }^{3}$ \\ College of IST ${ }^{1}$ \\ Drexel University \\ Philadelphia, USA \\ aleksarc@drexel.edu \\ Emergency Trauma and Burn Services ${ }^{3}$ \\ Children's National Medical Center \\ Washington, USA \\ rburd@childrensnational.org
}

\begin{abstract}
We conducted a study in a pediatric trauma center to elicit design requirements for the TraumaPen system-a mixed paper-digital interface using a digital pen and a wall display-to support situation awareness during trauma resuscitation. In this paper, we describe the field research that informed the initial system prototype and then present findings from two studies in which the prototype was used to further explore the application area. Our results showed the potential for digital pen technology in supporting teamwork in the dynamic and safety-critical setting of the trauma bay, but also revealed several limitations of this technology. We conclude by discussing challenges and requirements for the use of paper-digital interfaces in assisting fast-paced, collaborative work processes.
\end{abstract}

Keywords-pen-based computing; healthcare; situation awareness; collocated teams; trauma resuscitation.

\section{INTRODUCTION}

Trauma remains the leading cause of death and disability in children and young adults worldwide. Because early care after a major injury has an important impact on the patient outcome, the initial management of injured patients in the emergency department (trauma resuscitation) must be efficient and effective. During resuscitation, an interdisciplinary team of medical specialists (trauma team) must identify and treat potentially life-threatening injuries, often with the need for a critical decision about once every minute [1]. Awareness of events in the room and their progression is thus essential to efficient trauma care. To maintain situation awareness-obtain and interpret information about the patient, the actions of other team members, and the environment, and then use this information to predict changes in each-team members must attend to concurrent tasks, changing plans and personnel, and variable patient status. Information sharing is now only minimally supported by technology and trauma teams primarily rely on collective memory and verbal communication [2]. Furthermore, verbally reported information is often inaudible and inaccurately transmitted to other team members [3]. Although patient status and treatment plans are recorded in real time on a paper flowsheet by the nurse recorder, this record serves an archival role and offers minimal support for real-time work [4]. We believe that the role of the flowsheet can be expanded to improve information use during resuscitation.

Our long-term research goal is to develop information and communications technologies to improve situation awareness during dynamic safety-critical teamwork, such as trauma resuscitation. To elicit design requirements for such a system, we developed a prototype called TraumaPen and evaluated its feasibility in supporting trauma teamwork. We used a digital pen for real-time data capture and a large wall display for realtime data presentation. In doing so, we exploited the familiarity of current, low-tech documentation techniques in the trauma bay to introduce computational and communication mechanisms to improve information accessibility for members of the trauma team. The prototype design was based on findings from in-depth field studies at two US Level 1 (highest) trauma centers and followed an iterative design approach.

In this paper, we describe our requirements gathering and analysis process using the TraumaPen prototype and the findings from two studies in which the prototype was used to further explore the application area. Design solutions proposed here are initial steps toward reaching our long-term research goal. Introducing information technology (IT) in a domain such as trauma resuscitation is a complex problem that may take years to solve. Before we invest more resources into the system design and development, we need to assess if coupling digital pens with wall displays is feasible. We believe that researchers and practitioners will benefit from our initial results. Our iterative design and evaluation process revealed several design challenges for trauma resuscitation domain and showed that some claims made about digital pen technology in other medical settings are not transferable to the trauma bay. We discuss these challenges as well as insights about the use of information in time-critical work that need to be addressed in system design. The key contributions of this paper are:

- A prototype system to support situation awareness, designed and evaluated iteratively with involvement of trauma team members.

- Identified requirements and challenges for technologies to support situation awareness in a domain with rapid information acquisition and processing.

\section{A. Digital Pen Technology and Large Wall Displays}

Despite increasing use of advanced technologies in healthcare $[5][6][7]$, trauma resuscitation remains one of the few medical settings with minimal IT support, primarily depending on paper artifacts. The continued use of paper is a barrier to using real-time digital information processing for 

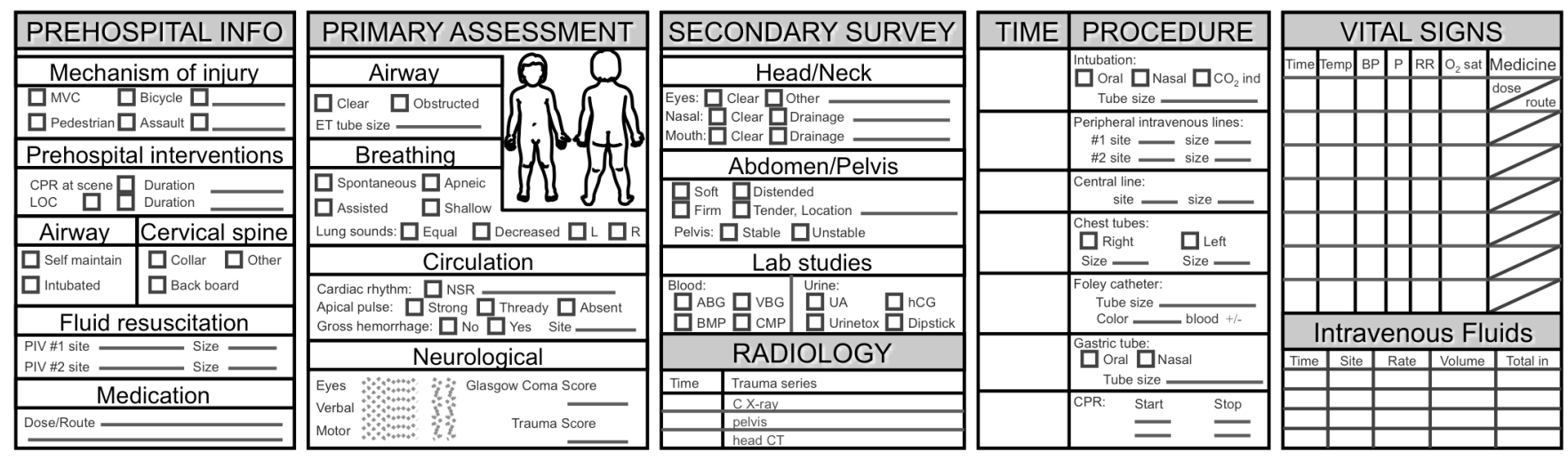

Figure 1. Paper-based trauma flowsheet.

improving patient care and reducing medical errors. Several attempts have been made to introduce IT in resuscitation areas, but they have not yet yielded feasible solutions [1]. One of the key reasons cited for the lack of success is the challenge of capturing and manually entering data from diverse sources.

Recent technological advances have begun to bridge the paper-digital gap. Rather than replacing paper with digital devices, new approaches have focused on augmenting paper and enabling connections between paper documents and information systems [8]. One such approach uses a digital pen as an input device that captures the location and time of pen marks for digital analysis. Digital pen technology retains the affordances of paper while allowing users to exploit digital interfaces in domains ranging from text processing [9] to military operations [10]. Digital pens have also been studied in medical settings as a tool for supporting speech and autism therapy [11][12], and as a method for supporting archiving [13][14], but have not been evaluated yet as a tool to aid highrisk medical collaborative work.

Large displays have been used to support situation awareness, task coordination, collaboration and information sharing [15][16][17]. Bardram et al. [5] deployed large interactive displays in a surgical ward to support spatial, temporal, and social awareness. Others have examined lowtech solutions such as whiteboards [18][19]. These studies have shown that status boards and wall displays can help support work that is distributed over people, time and space by facilitating task coordination and management, resource planning and tracking, synchronous and asynchronous communication, and problem solving.

Findings from this previous work led us to consider the potential of coupling digital pens with wall displays to support situation awareness during trauma resuscitation. Digital pens have been studied in some medical settings, yet its feasibility has not been evaluated in unpredictable settings such as resuscitation areas. Similarly, digital pens have been used for translating paper-based interactions onto large interactive whiteboards [20]. Yet, they have not been studied for the purposes of supporting situation awareness through real-time data presentation. Coupling digital pens with wall displays is a novel and potentially effective mechanism for supporting teamwork in work settings characterized by rapid information acquisition and processing.

\section{FROM FIELD RESEARH TO DESIGN REQUIREMENTS}

Our initial design of the TraumaPen system was based on findings from in-depth field studies conducted over the past four years at two US Level 1 trauma centers-an urban teaching hospital in the Northeast and an urban pediatric teaching hospital in the mid-Atlantic region. Both centers have similar trauma team composition and follow the standard protocol for patient evaluation and treatment [21]. A trauma team typically consists of an attending surgeon, a team leader (a surgical fellow or a senior resident), a physician doer (a junior resident), an anesthesiologist, a respiratory technician, a primary nurse, a medication nurse, a technician, and a nurse recorder. Patient evaluation protocol consists of a rapid primary survey of major physiological systems (Airway, Breathing, Circulation, and Disability), detailed secondary survey, and the initiation of definitive care. The two centers treat over 1,000 trauma patients per year each, most with injuries from car accidents, falls, and gunshot or stab wounds.

\section{A. Observed Problems and Preliminary Design Requirements}

Below we highlight key observations from our field studies that motivated and informed the initial design of the TraumaPen system. Detailed descriptions of methods and findings are published elsewhere [2][4][22].

\section{1) Information Overload}

Our studies identified the most frequently needed information types and showed how the use of information changed over the course of resuscitation. Trauma teams manage large amounts of information about patient status and team activities in a short time period (on average 20 to 30 minutes). Our analysis of team members' inquiries revealed over 60 different types of information needed in a typical event. The most frequent information included patient demographics, medical history, details about the mechanism of injury, vital signs, status of major physiological systems, and details about treatments and equipment used. Some information was needed at all times, while the need for other information faded over time. For example, mechanism of injury was important for making the initial decision about the patient treatment; airway status was needed during initial evaluation; age and weight were needed throughout the event to determine medication and fluid dosage. This finding led us to consider displaying only the most frequently sought information. 


\section{2) Reliance on Communication and Collective Memory}

Our studies also pointed to the need for external memory aids to support rapid acquisition and processing of information needed for decision making. Team activities and patient conditions are conveyed verbally and recorded on a paper flowsheet to facilitate subsequent medical care and post-event team evaluation, but not real-time decision-making (Figure 1). We found that team leaders seldom look up the flowsheet information to make decisions. Leaders reported that approaching the recorders' desk and finding information in the densely populated flowsheet is inconvenient and takes time. Because there are no mechanisms by which patient information is currently accrued to allow for rapid integration and analysis of patient data, trauma leaders mainly rely on verbal communication and collective memory for information access. Verbal reports, however, are often unreliable as team members forget to report their status. More important, dynamic information, such as past vital signs, or the amount of fluid and medications already administered, cannot be inferred by looking at the patient-bed area. We believed that displaying critical patient information using peripheral, large wall displays could facilitate information flow from providers to seekers, while also enabling quick information absorption at a minimum interference with the team's focus of attention - the patient. Displaying the entire flowsheet as-is would only clutter the display and make the information hard to access.

\section{3) Supportive Roles of the Recorder and the Flowsheet}

Furthermore, our studies suggested augmenting the role of the nurse recorder who, in addition to archiving, helps manage the resuscitation process by providing feedback to the team. To ensure accurate documentation of the large number of evaluation and treatment steps, the recording task is assigned to an experienced nurse who records the information on a paper flowsheet. The recording task is demanding and requires simultaneous attention to several information sources. Despite the challenges, we noticed that the flowsheet provided limited, but important support for trauma teamwork. The multiple-page flowsheet was usually spread open across a table, allowing easy navigation between sections and rapid data entry. The document also helped the recorder manage the process by allowing quick detection of missing information. Missing items usually implied skipped tasks and recorder's requests for these items often prompted the team to comply with the protocol.

Based on these findings, we concluded that the recorder could serve as a proxy for displaying up-to-date information for the team as she performs real-time documentation. The recorder already captures information from the environment, some of which is difficult to capture using automated techniques such as radio frequency identification (RFID), computer vision or speech recognition. Although used successfully in some environments, these technologies are not ready yet for the noisy, crowded and dynamic setting of the trauma bay. For instance, patient findings obtained through palpation are verbally reported and then manually recorded; speech recognition capture is currently not feasible.

Finally, the dynamic nature of trauma teamwork and a multitude of information sources and types suggested that complete and accurate documentation using computer data entry interfaces would be difficult. Although appealing as a solution, electronic medical record systems and tablet PCs have been found to slow down work processes in medical and other time-critical settings [10][23]. Additional challenge includes interacting with computers while having hands and eyes busy with the main task of patient care. These findings led us to explore the ways in which the paper and digital world could be bridged to support situation awareness while preserving the benefits of using pen and paper.

\section{TraumaPen System Prototype}

The TraumaPen system consists of two components: a digital pen and a large wall display. The purpose of the digital pen is to capture and store recorded information for presentation on the wall display, and for archiving. The purpose of the wall display is to present a set of recorded items in real time, as the recorder captures information on paper.

The system is based on the Anoto DP-201 digital pen that interfaces with the flowsheet. The flowsheet is printed on standard paper layered with the Anoto dot-pattern that enables the pen to capture user's writing. The software is based on the iPaper/iServer framework for capturing and processing paperbased information [24], and a novel toolkit for real-time data display; it runs on both Microsoft Windows and Mac OS X, and can be deployed on desktop computers or laptops. Selected data fields on the flowsheet were made "interactive" based on the information needs assessment from our field research. Writing or tapping into these fields with the digital pen results in real-time displaying of the entered information on the wall display. Information is now recorded using either checkboxes or freeform text (Figure 1). Our system supports both input modes: checkboxes are mapped directly onto the corresponding choices and stored as part of the TraumaPen data model, while freeform text is first processed by Intelligent Character Recognition software and then mapped onto corresponding entries in the data model. The data from the pen is transmitted wirelessly (over Bluetooth) to a computer that can be connected to any large monitor for immediate processing and real-time display.

\section{A. Interactive Display for Supporting Situation Awareness}

The wall display (Figure 2(a)) was designed to show the most frequently sought information by trauma team members, as found during field research. This initial design of the display was also screened by collaborating physicians to ensure that the displayed information is consistent with resuscitation protocol. The display included the following information:

The very top section of the display in Figure 2(a) includes patient demographics such as age and weight. As we have found in our field studies, demographic information is needed throughout the event for administering fluid or medications, and interpreting vital signs.

The middle section presents patient status and findings from physiological evaluations during different stages of the primary survey. For example, the finding "breath sounds equal bilaterally" from step $B$ (Breathing) is shown by green-colored lungs. Given the limited screen real estate and to enable quick identification and absorption of the critical information, we used graphical representation and color-coding for displaying primary survey findings. 

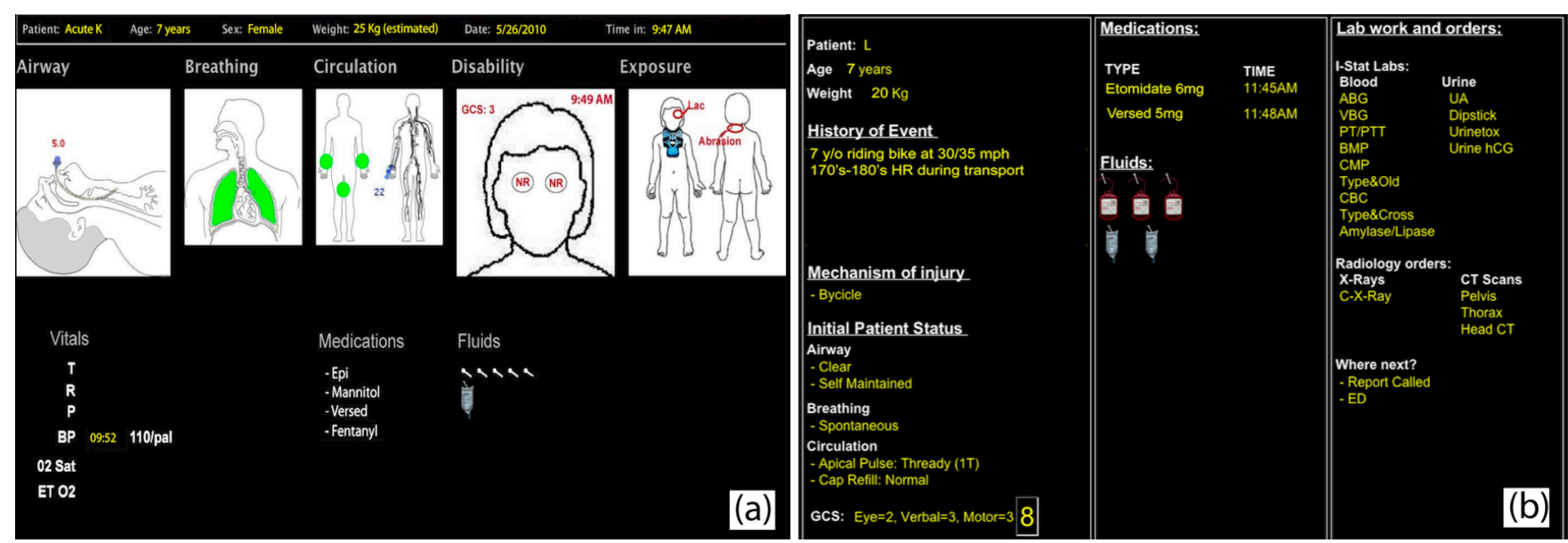

Figure 2. Two versions of the TraumaPen display design. (a) Initial display design based on findings from field research.

(b) Redesigned display based on focus-group discussions with trauma team members.

The bottom section presents vital signs (temperature [T], respiratory rate $[\mathrm{R}]$, heart rate $[\mathrm{P}]$, blood pressure $[\mathrm{BP}]$, oxygen saturation $\left[\mathrm{O}_{2} \mathrm{Sat}\right]$ and exhaled $\mathrm{CO}_{2}\left[\mathrm{ETO}_{2}\right]$ ), administered fluids, and administered medications. For each vital sign, we displayed the latest value and the time it was recorded; for each administered medication, we displayed medication type; and, for each bag of administered fluid (including blood), we displayed an icon of a fluid bag.

\section{Prototype Evaluation in the Trauma Bay}

To explore how coupling digital pens with wall displays may support situation awareness of trauma teams, we evaluated the TraumaPen prototype in two studies using a series of focus groups and simulation sessions with trauma team members. Because our main goal was to assess the system feasibility, our studies and subsequent design iterations focused on the displayed content and the use of the system. (We will focus on the details of graphical display for optimal team performance in future work). Our research questions were as follows:

RQ1: To what extent will the added task of documenting for real-time display affect the recorder's work patterns?

RQ2: To what extent will the current documentation practices affect real-time data presentation?

RQ3: To what extent will the display support situation awareness during resuscitation?

RQ4: Will the character recognition work well for real-time data capture and presentation in the trauma bay?

To conduct evaluation studies, we returned to our second research site-pediatric trauma center in the mid-Atlantic region. Both studies were approved by the hospital's ethics committee as an exempt research protocol. Below we describe methods and present findings from both studies.

\section{A. Study 1: Initial Prototype Evaluation}

\section{1) Methods}

We first evaluated the TraumaPen prototype with trauma nurses and team leaders using focus groups: two with fourteen nurses (seven in each) and one with four senior surgical residents. We started with nurses and team leaders because we saw them as the primary users of our system: nurse recorders use the digital pen to capture information for real-time display and team leaders use the display for information access and decision making. We used homogeneous focus groups to allow for in-depth discussion of design issues. Because of differing places of each group in the medical hierarchy, we felt we would obtain better feedback using this approach.

Each focus group lasted an hour and involved playing a 5minute video simulating the use of the system at the beginning of each session, followed by discussion. The video told a story about an injured child brought to the center and followed a typical resuscitation scenario including pre-hospital report, evaluation, and treatments. We focused on presenting changes on the interactive display as the scenario evolved and as the nurse documented the events. A video-in-video showed a flowsheet close-up with a hand recording the information using the digital pen. This strategy was an effective design approach because it helped trauma team members match their domain knowledge with a future design of the system in the context of their work. Group discussions primarily focused on answering the first three research questions. All discussions were audiotaped and transcribed. To analyze the data, we used an open coding technique to identify recurring themes and issues about the system and its feasibility. We next present our findings organized by the two user groups.

\section{2) Findings from Focus Groups with Nurses}

Trauma nurses thought that digital pen technology was appropriate because it seemed consistent with their current work practices. Displaying evaluation steps and findings was perceived as beneficial. Nurses felt that this information would help the team know immediately what evaluation steps have been completed: "It gives everybody a visual, it helps us make sure we don't miss anything and keeps us on track, like, we haven't checked pupils, ok let's check them now." (Nurse3)

The system was also perceived useful in ensuring that the information is documented accurately:

"This is going to make people more sensitive to making sure they document things appropriately and update vitals regularly. If a surgical resident looks up and see things we 
documented inappropriately, he can then tell us 'hey, that wasn't what I found or reported." ' (Nurse7)

Information about administered medications and fluid was also found helpful. The nurses felt that times for each administered medication should be displayed to enable easier tracking and timing of subsequent doses. They also suggested including details about mechanism of injury to accommodate team members joining in late.

The nurses, however, expressed two main concerns about the TraumaPen system. First, they felt that displayed information would not be up-to-date as they often alternate between documenting different data elements:

"I would go back and forth, and this [mechanism of injury], a lot of times I don't even write the history until I actually know what happened... by the time paramedics finish their report, I am already recording my first set of vitals, so I'll just remember the report, focus on what's going on, and then go back and write down the report." (Nurse2)

Second, there was a concern about displaying changes in the patient status. Because the status may change rapidly, it is important to make these changes visible on the display. Changes in patient condition after the initial assessment had been completed are now documented in progress notes, a freeform text section in the flowsheet where nurses present a narrative of events. The structure of progress notes vary from nurse to nurse, which may pose major challenges for detecting and recognizing updates in patient conditions.

\section{3) Findings from Focus Groups with Trauma Leaders}

In contrast to nurses, surgical residents thought that having findings from primary survey displayed graphically would not be useful. They explained that this information could be easily obtained by just looking at the patient: "Seeing a tube drawn on the face and looking down on the patient and seeing a tube in the mouth is about the same." (Resident2)

They instead suggested putting emphasis on patient demographics, mechanism of injury and initial evaluation findings to prevent interruptions by latecomers and others who missed the pre-hospital report, which is important in making the initial decision about the patient treatment:

"Patient name, mechanism of injury, vital signs in the field and on arrival, GCS score and then initial findings from $A B C D$ is about all you will need on that board that anybody who walks in that room will need to know." (Resident3)

The residents also found information about medications and fluids useful. They explained that tracking administered medications and fluid is often difficult due to the noise and poor communication in the trauma bay:

"If you are a trauma leader, all you need up there is age, mechanism, so that people can figure that out, and medications and fluids that have been given, like a tally... having an active record of how much fluid has been given so that you can just look up and see, and see what medications have been given." (Resident1)
Similar to nurses, team leaders expressed a concern with the system's ability to display changes in patient status:

"Updating information on the board is important because things change rapidly, especially with kids, they will compensate for as long as they can, but they can suddenly crash, and if the board still shows that everything is fine, that is going to be a problem." (Resident3)

Finally, the residents thought that the display should present patient management information including blood work orders, $\mathrm{x}$-ray and CT-scan orders. This information is needed by staff who place orders and frequently interrupt surgical leaders when asking about patient management:

"Other people need to know what x-rays and labs need to be ordered, because one of the main things that I have issues with is that you need to tell your orders to five people, but if you tell it to the charge nurse and she writes down 'basic trauma labs' and boom, it says 'basic labs' up there, so anyone can walk in and see that's the labs they want and then they start working on it as opposed to us having to tell repeatedly several times. " (Resident2)

\section{B. Design Iterations Based on User Feedback}

Using the findings from focus groups, we redesigned the TraumaPen display as follows (Figure 2(b)). First, we removed graphical representation of patient findings during primary survey given the perceived difficulty of automatic detection of and inference about changes in the patient status from freeform progress notes. Detecting and recognizing updates in patient conditions from freeform text will be part of our future work. Our display redesign still included findings from initial patient evaluation because they are captured using checkboxes and easily transferable to the display. We also included information about the mechanism of injury (captured by checkboxes) and the event history (captured by freeform text). Together with initial evaluation findings, this information comprises the left pane in Figure 2(b). The middle pane contains information about medications and fluids. Following nurses' and residents' suggestions, we included the time and dosage for each administered medication. The bottom section of the middle pane is intended for future trending of vital sign data. The right pane shows lab, x-ray and CT-scan orders.

\section{Study 2: Simulation Evaluation}

We conducted a follow-up study with a redesigned display to further explore the application area and gather additional design requirements. The study involved training sessions with nurses and evaluating the system in two simulation scenarios.

\section{1) Training Sessions: Methods}

We first conducted individual training sessions with six trauma nurses. The primary goal of these sessions was to prepare nurses for simulations by training them on the TraumaPen system. The sessions provided an opportunity to further assess the extent to which using the system impacts nurses' work and how current documentation practices may affect real-time data presentation. We also ran initial tests on character recognition using nurses' handwriting. Each training session lasted 30 minutes and consisted of: (a) a brief introduction to the system, (b) simulation of the documentation 


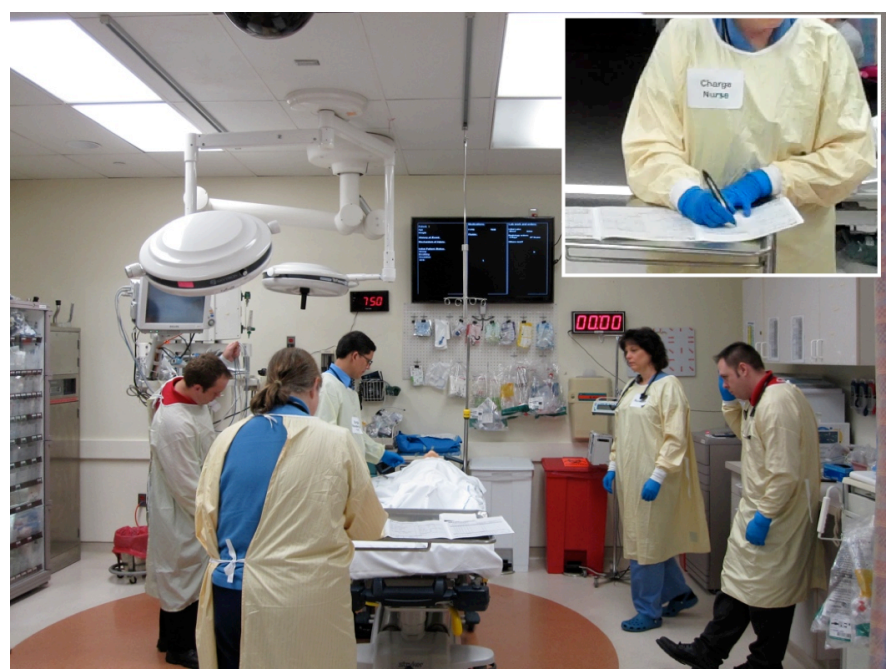

Figure 3: Simulation setting, arrangement of trauma team members, digital pen in use (upper right corner) and the TraumaPen display. Second display

(not shown) is mounted on the wall opposite to the first display.

process by using the digital pen and by watching a 10-minute video of a real trauma simulation, and, (c) discussion about nurses' experiences. The nurses were seated at the desk, with the TraumaPen display positioned in front of them and simulation video projected on the wall on their right-hand side. During the recording activity, we observed nurses' interactions with the pen and whether they were looking at the display. The current design of the flowsheet was not altered for the study. The only modification was overlaying the Anoto dot-pattern on the document. Each session was audiotaped and transcribed. We again used the open coding technique to identify major themes about the system's use and its impact on nurses' work.

\section{2) Training Sessions: Findings}

All six nurses reported that using the digital pen felt like using a traditional pen. As one of the nurses commented: "It works as normal pen, the documentation part isn't going to be different if we can use it like we normally do." One nurse, however, noticed that the digital pen might affect her charting because "you'll have to be more careful about not just circling something, it can look OK on paper but off on the screen."

One of our concerns was how distracting the display would be for nurses as they performed the documentation task. Our expectation was that nurses would continue to focus on the documentation and would glance at the display only occasionally or not at all. During training sessions, nurses emphasized that documentation requires their absolute attention and does not leave much time for looking at the display:

"I didn't look up because things are moving so fast in there that I am constantly keeping up on [the form]. I was so focused on listening and writing, but if I were in a real trauma I would have to attend to million things at once and I probably wouldn't have a chance to look up until the survey was over."

We noticed however that one nurse occasionally glanced at the display. When asked about the reasons for looking at it, this nurse commented, "I was interested if it was picking up what I was writing." This finding revealed that nurses might look up to check for correctness of the displayed information.
Training sessions confirmed the issue of potential delays in displaying up-to-date information that was first raised during focus groups. Still, we observed that some information is recorded at the time it is reported. Examples include medications, a neurological scale for assessing consciousness, and vital signs. Nurses pointed out that despite variability in their work, documentation depends mostly on what they hear or see. Communication failures and skipped evaluation steps may impact when the information is documented.

Finally, the training sessions pointed to problems with handwriting recognition and information accuracy. As all six nurses engaged in recording activity, we observed that the system was not recognizing their handwriting properly. Handwriting recognition raised concerns about accuracy of the displayed information:

"If for medications it shows 'phantoms' instead of 'etomidate' or '16mg' but we actually gave '6,' it's a big deal... especially things like kilos, we'll have to check because if it's inaccurate up there, then that can be a real problem."

\section{3) Design Iterations Based on User Feedback}

Based on our findings from training sessions we decided to modify the system before doing simulations with trauma teams. Because handwriting recognition emerged as the most prominent issue, we replaced recognition with capturing and displaying an image of handwritten text for all active, freeform fields. We felt this modification would improve accuracy of the displayed information and help trauma teams better perceive the potential of our system in supporting situation awareness.

\section{4) Simulation Sessions: Methods}

We further evaluated the TraumaPen prototype in the context of simulated trauma resuscitation, with a total of 13 trauma team members. Simulations have commonly been used to evaluate team performance. While realizing that a patient simulator is being used, participants frequently "suspend their disbelief" and perform in a manner similar to actual clinical scenarios [25]. The first simulation involved an attending surgeon, a junior surgical resident, and five nurses, two of whom participated in earlier training sessions. The second simulation involved an attending surgeon, two pediatric nurse practitioners who often assume a physician doer role, one senior- and one junior surgical resident, and one TraumaPentrained nurse. Both sessions followed the same clinical scenario and lasted about 10 minutes each. Our goals here were to obtain additional feedback using heterogeneous focus groups in the semi-real environment of the trauma bay (Figure 3).

To display awareness information, we connected our system to two 42" wall mounted-monitors visible to team members at the head and foot of the bed. Both displays showed the same information. Sessions were videotaped to track who among the participants looked at the display and when during simulation. The nurse recorders were given the digital pen and Anoto-patterned flowsheets. At the beginning of each session, we briefly introduced the system. Upon completion of each simulation scenario, we conducted a group discussion with participants. Discussions were audiotaped, transcribed, and analyzed in the same manner as reported earlier. 


\section{5) Simulation Sessions: Findings}

We observed that team members frequently glanced at the display during the simulation sessions. Glancing at the display mostly occurred in the first five minutes, while the teams performed primary survey, patient intubation, and fluid administration. For example, in the first session, when the team leader ordered a liter of fluid, the primary nurse first looked at the monitor and then turned to the recorder, inquiring, "I am sorry, I can't read his weight, what is it?" Or, in the second session, when the recorder asked about the fluid rate, all team members glanced at the monitor. The recorders did not look at the display; they were busy documenting and communicating with other team members about patient information.

In current practice, obtaining patient information requires looking around or querying the recorder, or the person who owns the information. Our preliminary data from simulations showed promise in using information displays for faster access to patient information. A quick glance at a well-designed display takes less time than engaging in verbal exchange with another team member.

Simulation sessions also confirmed some of the limitations of the TraumaPen system. Although we replaced character recognition with an image of handwriting, we observed that team members had trouble reading the handwritten text in the images. First, text in the images was small and illegible from distance. Second, readability of text now depended on readability of the recorder's handwriting. Although both recorders had neat handwriting, the images were difficult to read and raised concerns about the ability to capture and effectively display freeform text information.

\section{DISCUSSION}

Findings from evaluation studies in a pediatric trauma center confirmed that there is a need to support situation awareness during trauma resuscitation. Through an iterative design and evaluation process that involved trauma team members, we were able to better understand trauma teams' information needs as well as documentation practices that are critical to reliable functioning of the TraumaPen system. Although team members' comments and interactions with the TraumaPen showed promise for digital pen technology in this setting, the studies also revealed several challenges for use in such a complex and safety-critical domain.

\section{A. Added Task of Documenting for Real-Time Display}

Our first research question was to what extent would the added task of documenting for real-time display affect the recorder's work patterns. Results from training sessions and simulations showed that nurse recorders primarily focused on documenting and rarely looked at the display. Nurses also felt that the digital pen did not affect their current way of recording information. These findings suggest that having the recorder as a proxy for displaying up-to-date patient information can be an effective way of supporting trauma teamwork.

Still, our studies raised an important issue related to nurses' use of the system. As we observed during training sessions, nurses might need to look at the display frequently to check the correctness of displayed information. Because frequent glances at the display distract nurses from their primary task, the challenges of ensuring the correctness of information and its appropriate display should be addressed. One positive aspect of displaying recorded information for the whole team is that the task of checking for correctness is now distributed among team members. In addition to helping trauma teams maintain situation awareness, distributing the task of checking for correctness may also help improve the documentation process. Another solution could be adding some form of feedback or alerting mechanism to allow timely response to incorrect or inappropriately displayed information. As team members repeatedly emphasized, displaying incorrect information would make the system useless or detrimental.

\section{B. Real-Time Documentation vs. Real-Time Presentation}

Our evaluation studies revealed an apparent disconnect between real-time documentation and presentation. Some information is captured as observed or reported, while other information is held in memory and recorded at a later time. Although we initially believed that real-time documentation was predictable, our study uncovered this discrepancy by introducing a novel use of the recorded information: in addition to its traditional use for archiving, it was now being used to support situation awareness. We believe that this finding is valuable when considering other technologies in dynamic work settings. The order in which the information is documented may impact the design and should be considered. Even so, our findings showed that documentation process is not random. Some information types are recorded immediately and in the order they are received and can thus be displayed using the TraumaPen system. Our future work will further examine the prioritization of information documenting and identify information items that are suitable for digital pen technology.

The studies also showed the importance of allowing frequent updates or corrections of the displayed information. As reported by trauma team members, patient conditions change rapidly. For the system to be useful, it must present upto-date information and the time it was last updated. When a nurse makes a mistake on the flowsheet, he or she strikes the data through and writes the correct information next to it. Observed changes in the patient condition after the initial evaluation had been completed are now documented in freeform progress notes. Future prototypes of the TraumaPen should detect corrections on the flowsheet and automatically infer important information from progress notes that is pertinent to patient conditions.

\section{Differing Information Needs}

Our study suggests that supporting situation awareness in trauma resuscitation may require several displays for different information types and for different users. As seen from participants' comments, not all information is equally important to all team members. Information about patient findings, administered medications and fluids helps support situation awareness of decision-makers, while information about mechanism of injury or lab orders helps latecomers and supporting staff. Our findings also showed the differing views of nurses and surgical residents on the usefulness of graphical representation of physical assessment. As documenters, nurses viewed this information as a helpful tool in providing a quick overview of what tasks have been completed and what tasks 
were skipped. Conversely, trauma leaders perceived this information extraneous; being at the patient bedside and supervising the process, they are able to see the patient's current condition. Our future iterations of the TraumaPen system will involve other stakeholders and careful examination of differing information needs to ensure that the most critical needs of each role are addressed. We will also explore issues related to graphical layout to determine the best ways of visualizing data for rapid access and absorption.

\section{Handwriting Recognition in Dynamic Work Settings}

The last research question examined the extent to which information systems can rely on handwriting recognition in dynamic work settings such as trauma bay. Despite promising evidence [10], our findings showed that both the speed with which nurses document and the use of medical terms make character recognition challenging in this domain. Even our alternative solution of using an image of handwritten text showed only limited promise. Nurses' handwriting styles vary, posing challenges to presenting handwritten information.

Our findings also showed that not all fields on the flowsheet are optimally designed for real-time capture and display. The digital pen worked best for displaying items captured through checkboxes. To display handwritten information using the digital pen, both redesigning of the flowsheet and changing nurses' work practices may be needed. An alternative solution is to combine several techniques to capture information critical to decision making, such as RFID or computer vision technology. Although not yet mature for the crowded and noisy environment of the trauma bay, these automated techniques may be used for capturing some information types, e.g., medications or equipment.

\section{CONCLUSION}

We have designed, built and studied a prototype TraumaPen system to support situation awareness in the fastpaced setting of the trauma bay. Although our findings show the benefits and feasibility of our approach, they also highlight challenges in designing technology for the complex, safetycritical domain of trauma resuscitation. Using an incremental approach to system design, we identified important requirements for and challenges to introducing technologies in this domain. We realize that changes in practice may be needed to exploit technologies for supporting trauma teamwork. We believe that findings from this study will offer useful insights for supporting design work in other safety-critical domains.

\section{ACKNOWLEDGMENT}

This work is supported by NSF grant \#0915871. We thank the medical staff at the research site for their participation.

\section{REFERENCES}

[1] M. Fitzgerald, P. Cameron, C. Mackenzie, N. Farrow, P. Scicluna, R. Gocentas, et al., "Trauma resuscitation errors and computer-assisted decision support," Arch Surg., vol. 146, February 2011, pp. 218-225.

[2] A. Sarcevic, I. Marsic, M. E. Lesk, and R. S. Burd, "Transactive memory in trauma resuscitation," Proc. CSCW 2008, San Diego, California, USA: ACM Press 2008, pp. 215-224.

[3] E. A. Bergs, F. L. Rutten, T. Tadros, P. Krijnen, and I. B. Schipper, "Communication during trauma resuscitation: Do we know what is happening?" Injury, vol. 36, August 2005, pp. 905-11.
[4] A. Sarcevic, "Who's scribing? Documenting patient encounter during trauma resuscitation," Proc. CHI 2010, Atlanta, Georgia, USA: ACM Press 2010, pp. 1899-1908.

[5] J. E. Bardram, T. R. Hansen, and M. Soegaard, "AwareMedia: A shared interactive display supporting social, temporal, and spatial awareness in surgery," Proc. CSCW 2006, Banff, Alberta, Canada: ACM Press 2006, pp. 109-118.

[6] E. McLoughlin, D. O'Sullivan, and D. C. Wilson, "MEDIC: MobilE diagnosis for improved care," Proc. SAC 2006, Dijon, France: ACM Press 2006, pp. 204-208.

[7] J. Silva, M. Zamarripa, P. Strayer, J. Favela, and V. Gonzalez, "Empirical evaluation of a mobile application for assisting physicians in creating medical notes," Proc. AMCIS 2006, Acapulco, México, pp. 2673-2681.

[8] P. Luff, C. Heath, M. Norrie, B. Signer, and P. Herdman, "Only touching the surface: Creating affinities between digital content and paper," Proc. CSCW 2004, Chicago, Illinois, USA: ACM Press 2004, pp. 523-532.

[9] N. Weibel, A. Ispas, B. Signer, and M. C. Norrie, "PaperProof: A paperdigital proof-editing system," Ext. Abstracts CHI 2008, Florence, Italy: ACM Press 2008, pp. 2349-2354.

[10] P. R. Cohen and D. R. McGee, "Tangible multimodal interfaces for safety-critical applications," Communications of the ACM, vol. 47, 2004, pp. 41-46.

[11] A. M. Piper, N. Weibel, and J. D. Hollan, "Introducing multimodal paper-digital interfaces for speech-language therapy," Proc. ASSETS 2010, ACM Press, pp. 203-210.

[12] J. A. Kientz, G. R. Hayes, G. D. Abowd, and R. E. Grinter, "From the war room to the living room: Decision support for home-based therapy teams," Proc. CSCW 2006, ACM Press, pp. 209-218.

[13] C. Tang and S. Carpendale, "Supporting nurses' information flow by integrating paper and digital charting," Proc. ECSCW 2009, Vienna, Austria, pp. 43-62.

[14] P-Y. Yen and P. N. Gorman, "Usability testing of a digital pen and paper system in nursing documentation," Proc. AMIA 2005, pp. 844-848.

[15] P. Dourish, and V. Bellotti, "Awareness and coordination in shared workspaces," Proc. CSCW 1992, ACM Press 1992, pp. 107-114.

[16] R. Su, and B. Bailey, "Towards guidelines for positioning large displays in interactive workspaces," Proc. INTERACT 2005, pp. 337-349.

[17] S. Wilson, J. Galliers, and J. Fone, "Not all sharing is equal: The impact of a large display on small group collaborative work," Proc. CSCW 2006, Banff, Alberta, Canada: ACM Press 2006, pp. 25-28.

[18] H. J. Wong, M. Caesar, S. Bandali, J. Agnew, and H. Abrams, "Electronic inpatient whiteboards: Improving multidisciplinary communication and coordination of care," International Journal of Medical Informatics, vol. 78, 2009, pp. 239-247.

[19] Y. Xiao, C. Lasome, J. Moss, C. F. Mackenzie, and S. Faraj, "Cognitive properties of a whiteboard: A case study in a trauma centre," Proc. ECSCW 2001, Bonn, Germany, pp. 259-278.

[20] P. Brandl, M. Haller, J. Oberngruber, and C. Schafleitner, "Bridging the gap between printouts and digital whiteboard," Proc. AVI 2008, Napoli, Italy: ACM Press 2008, pp. 31-38.

[21] American College of Surgeons. Advanced Trauma Life Support $\mathbb{R}$ ( $A T L S(\mathbb{R}), 8$ th Edition. Chicago, IL, 2008.

[22] A. Sarcevic and R. S. Burd, "What's the story? Information needs of trauma teams," Proc. AMIA 2008, pp. 641-645.

[23] B. T. Karsh, M. B. Weinger, P. A. Abbott, and R. L. Wears, "Health information technology: fallacies and sober realities," J Am Med Inform Assoc., vol. 17, 2010, pp. 617-23.

[24] M. C. Norrie, B. Signer, and N. Weibel, "General framework for the rapid development of interactive paper applications," Proc. CoPADD 2006, pp. 9-12.

[25] G. B. Nackman, M. Bermann, and J. S. Hammond, "Effective use of human simulators in surgical education," J Surg Research, vol. 115, December 2003, pp. 214-218. 